\title{
Student activity: verification on Malus's law of polarization at low cost
}

\author{
Shahrul Kadri, David Chong, Bor Wei, Rosly Jaafar
}

Shahrul Kadri, David Chong, Bor Wei, Rosly Jaafar, "Student activity: verification on Malus's law of polarization at low cost," Proc. SPIE 9289, 12th Education and Training in Optics and Photonics Conference, 92892A (17 July 2014); doi: 10.1117/12.2070727

SPIE Event: 12th Education and Training in Optics and Photonics Conference, 2013, Porto, Portugal 


\title{
Student Activity : Verification on Malus's Law of Polarization at Low Cost.
}

\author{
Shahrul Kadri*, David Chong Bor Wei and Rosly Jaafar \\ Physics Kit and Instrumentation Research Group (PhyKIR), Faculty of Science and Technology, \\ Universiti Pendidikan Sultan Idris, 35900 Tg Malim, Perak, MALAYSIA \\ Corresponding author : shahrul.kadri@fsmt.upsi.edu.my*
}

\begin{abstract}
Polarization is the property of electromagnetic waves that describes the orientation of their field oscillation. Students face hard problem to visualize the important of the polarization in daily life. In common practice, students are taught with the help of sketch diagram. In this project, we describe both quantitative and qualitative ways of experimenting polarization using very low cost and easily available material: polarized sunglasses, PVC tubes, light-dependent resistors, LED, etc. With this set, the experiment of the Malus's Law verification of light polarization can be done without the need of expensive optical detector for quantitative measurement. Students will develop their own simple optical sensor if the set is developed as a project. This set of experiment integrates the concept of basic electricity. Therefore, students acquires the practical knowledge of electricity and optics in the easy way.
\end{abstract}

Keywords: Malus's Law, polarization, Student activity, low cost experiment

\subsection{Scenario}

\section{INTRODUCTION}

Polarization can be simplified to just means 'orientation'. Polarization is the property of certain waves that describes the orientation of their oscillation. The topic is taught at pre-University level such as in Sijil Tinggi Pelajaran Malaysia (STPM) (Item 23.7) and Cambridge International A \& AS Level Physics (Item 15). [1, 2]

Students face hard problem to visualize the important of the polarization in daily life. In common practice, students are taught with the help of sketch diagram. In this paper, we describe a student activity kit that will enable student to explore light polarization which is not just qualitatively but also quantitatively according to Malus's Law. This simple activity involves the construction of polarizer tube using two Polaroid lenses cut into a Polyvinyl Chloride (PVC) pipe. The construction of a simple circuit of light intensity detector using light dependent resistor (LDR) is also described. The materials used in this activity are cheap and affordable where it only cost less than MYR 30 (EUR7). Low cost experiment is essential especially in the developing countries where resources in teaching and learning is very limited [3].

\subsection{Malus' Law}

For unpolarized light, the electric and magnetic field components of light waves oscillate in random directions having more than one plane. In our modern life, we could have unnoticed polarized light from some electronic devices, such as calculators, computer screens and digital watches. The liquid crystal displays of these electronic devices emits energy in the form of polarized light. For most LCD screens, the polarized light is tilted $45^{\circ}$ from the horizontal. When we observed the LCD display of these electronic devices through a polarizer, the intensity of polarized light decreases and increases as we rotate the polarizer. The intensity of polarized light will become minimum when the polarization axis of the polarizer is perpendicular with the emitted polarized light. With this knowledge, polarization plane or axes of an unknown polarizer could be easily determined by observing the linear polarized light from these electronic devices.

According to Malus's Law, when completely plane polarized light is incident on the analyzer (a polarizer), the intensity $I$ of the light transmitted by the analyzer is directly proportional to the square of the cosine of angle between the polarization axis of the analyzer and the polarization axis of the polarized light, $\theta$.

12th Education and Training in Optics and Photonics Conference, edited by

Manuel F. P. C. Martins Costa, Mourad Zghal, Proc. of SPIE Vol. 9289, 92892A

(C) 2014 SPIE, OSA, IEEE, ICO · doi: 10.1117/12.2070727

Proc. of SPIE Vol. 9289 92892A-1 
The law can be stated mathematically as

$$
I=I_{o} \cos ^{2} \theta
$$

where $I$ is the light intensity after analyzer, $I_{o}$ is the light intensity before polarizer and $\theta$ is the angle between polarization axis of polarizer and analyzer [4].

The apparatus arrangement for testing Malus's law is illustrated as in Figure 1.

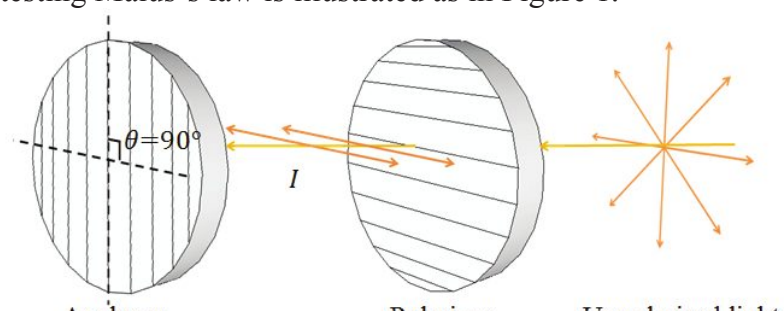

(a)

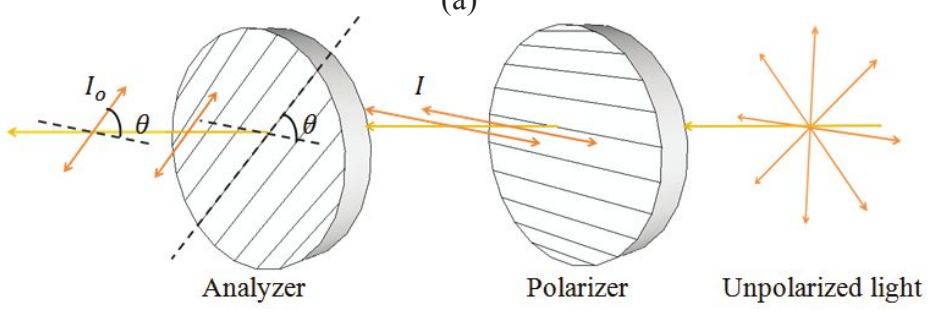

(b)

Figure 1 : Polarization of light after passing through 2 polarizers. (a) Cross-polarizer (b) Axes of polarization at certain angle.

By rotating the analyzer, students can observe the changes of light intensity in term of brightness that passing through the two polarizers (analyzer and polarizer). The variation of intensity as the function of angle $\theta$ is shown in Figure 10. The normalized intensity is the ratio of the output and input light intensities $I / I_{o}$.

\subsection{Raw Materials}

\subsection{DEVELOPMENT}

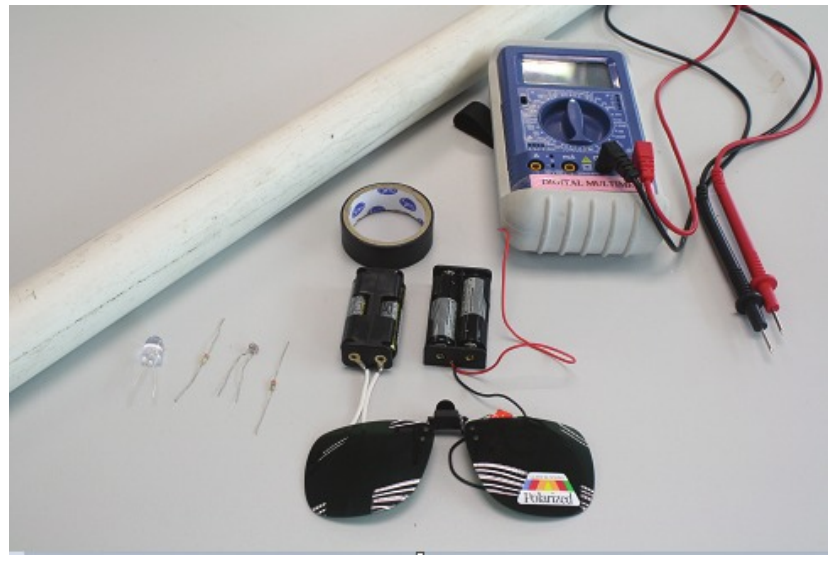

Figure 2: Components or raw materials in construction of the model.

The activities guide for experiment described in this paper is provided in the Appendix. A polarizer tube is constructed using two Polaroid lenses from a pair of sunglasses and PVC pipe. The first and second polaroid lenses acts a polarizer and an analyzer. Light source and a light intensity detector caps are built and attached to the both ends of the polarizer 
tube. The light source section emits unpolarized light at which it consists of a circuit comprises of two 1.5 volts batteries, a Light Emitting Diode (LED) and $120 \Omega$ resistor. While the light intensity detector consists of a circuit that connects light dependent resistor (LDR) to a pair of batteries $(1.5 \mathrm{~V}), 2.2 \mathrm{M} \Omega$ resistor and a digital multimeter. All these components were shown in Figure 3.

\subsection{Cost of Raw Materials}

The total estimated cost of the raw materials is shown in Table 1.

Table 1: Cost of Raw Materials.

\begin{tabular}{ll}
\hline Polarizer tube & MYR7.00 \\
\hline Polarized sunglasses & MYR 5.00 \\
\hline PVC tube $50 \mathrm{~cm}$ & MYR 2.00 \\
\hline Light source & MYR 1.50 \\
\hline 2 Batteries $1.5 \mathrm{~V}$ & MYR 1.00 \\
\hline 1 Batteries holder & MYR 0.20 \\
\hline LED & \\
\hline Light Detector $\Omega$ resistor & MYR 0.80 \\
\hline LDR & MYR 2.00 \\
\hline 2 Batteries 1.5 V & MYR 1.50 \\
\hline 1 Batteries holder & MYR 0.2 \\
\hline 2.2 M $\Omega$ resistor & AVailable in physics lab \\
\hline Digital OR Analog Multimeter/Voltmeter & \\
\hline Others & MYR 3.00 \\
\hline $\begin{array}{l}\text { Masking tape, silicon gum, soldering iron at etc. } \\
\text { for cosmetic and connecting parts }\end{array}$ & MYR 24.20 (EUR6.21) \\
\hline Estimated cost & \\
\hline
\end{tabular}

\subsection{Assembly}

Four main sections of the polarizer tube were constructed by using the raw materials listed in Table 1. The arrangement of the assembled and ready apparatus is shown in figure 4 . The polarizer and analyzer can be freely rotatable clockwise and anticlockwise after being assembled.

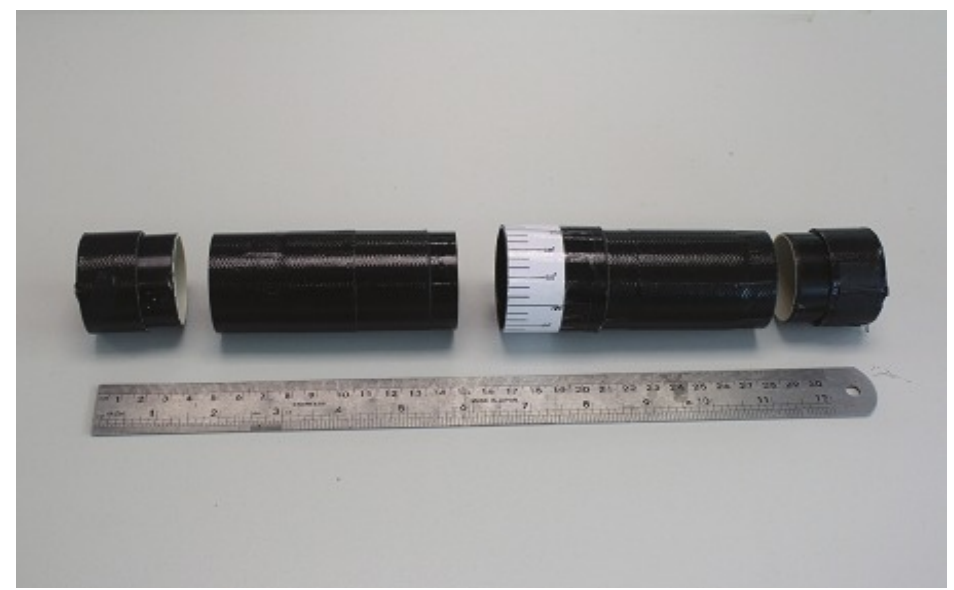

Figure 3: The assembled and ready polarizer tube made from raw materials in the Figure 3.

In order to determine the intensity of polarized light quantitatively, two simple electrical circuits are designed so that they could be connected at both of the ends of polarizer-analyzer arrangement. 


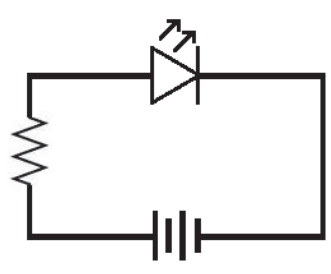

Figure 4: Light source circuit.

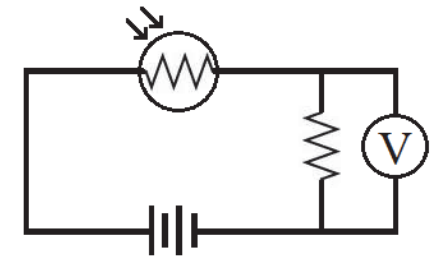

Figure 5: Light intensity detector.

The first circuit as in Figure 5 is used as the light source circuit while the second circuit functions as in Figure 6 is a light intensity detector. A light dependent resistor (LDR) is used as a sensing element to measure the intensity of polarized light, as the intensity of polarized light increased, the resistance of the LDR will decrease. Since the resistance of the fixed resistor remains the constant, the output voltage measured across the fixed resistor will increase. Thus, the output voltage measured is directly proportional to the intensity of polarized light. When a graph of output voltage, $V_{\text {out }}$ against the angle between the polarization axes of analyzer and polarizer, $\theta$ is plotted, the graph will have the pattern of Figure 2 according to Malus's Law.

\subsection{Polarization Axis of a Polarizer}

Our naked eyes can not distinguished between polarized and unpolarized light. However, student can notice the polarized light by observing emitted light from most electronic gadget display. Image displayed on the screen of a calculator or a computer screen or any LCD is observed through a polarizer (Figure 7). The variation of brightness is observed when the polarizer is slowly being rotated. The perpendicular axis of the polarizer is confirmed when the view is dark and parallel axis is determined when the view is bright (Figure 8).

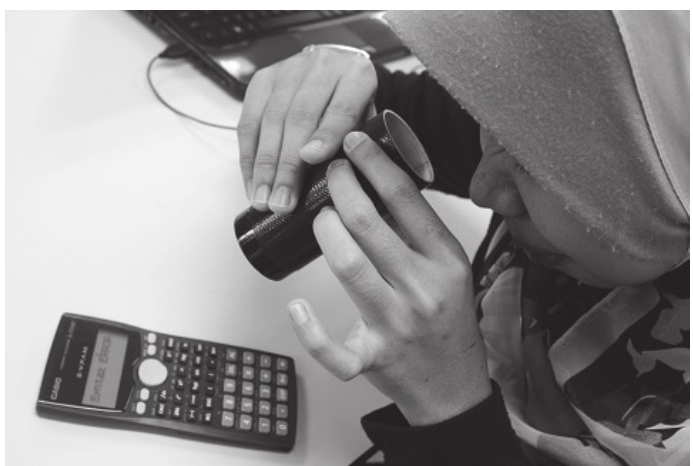

Figure 6: Observing LCD through the constructed polarizer

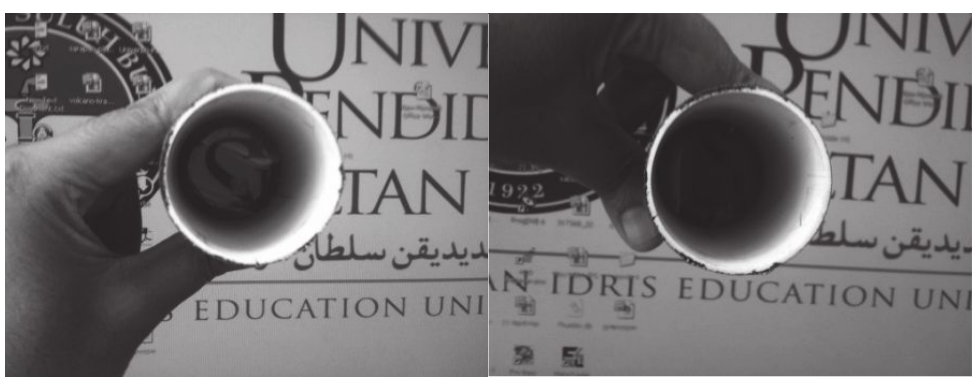

Figure 7: Determining the polarization direction of the polarizer. Left: The direction of light polarization is parallel to the polarizer. Right : The direction of light polarization is perpendicular to the polarizer, therefore the emitted light is blocked (dark). 


\subsection{Verifying Malus's Law Qualitatively}

The polarizer section and analyzer section were connected together. The light intensity of the unpolarized light source such as light from ceiling lamp or table lamp were observed while slowly rotating the analyzer $360^{\circ}$ relatively to the polarizer (Figure 9).

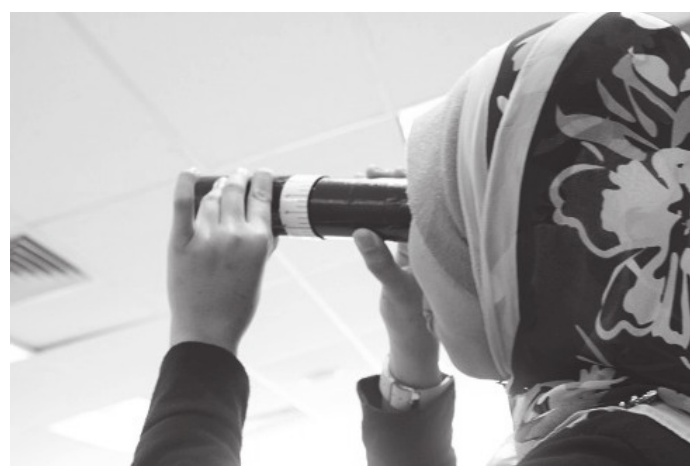

Figure 8: Observing the light intensity of unpolarized light source (room light) while slowly rotating the analyzer.

\subsection{Verifying Malus's Law Quantitatively}

All four sections of the polarizer tube were connected to each other in arrangement that has been described earlier in Figure 4. Both circuits (light source and detector) are connected to the batteries and a digital multimeter is connected to the voltage divider circuit to measure its output voltage, $V_{\text {out }}$ as shown in Figure 10.

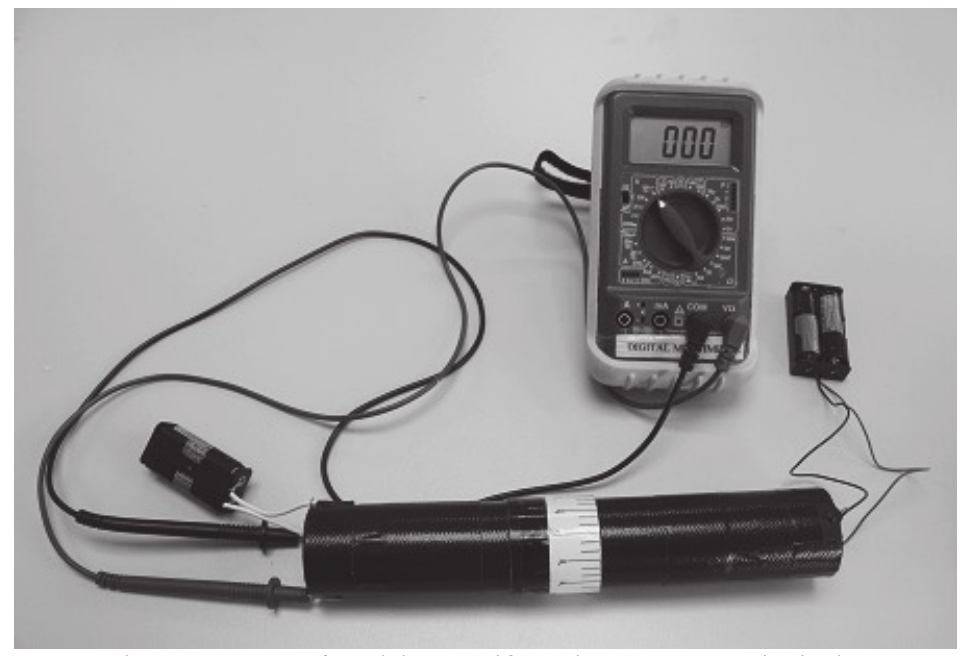

Figure 9: Set up of models to verify Malus's Law quantitatively.

The analyzer was rotated $10^{\circ}$ ach time until $360^{\circ}$ were completed. At each $10^{\circ}$, the output voltage of were recorded and analysis were carried on the graph generated from the data collected.

\subsection{DISCUSSION}

\subsection{Qualitative And Quantitative Measurement}

The polarizer that set on to test were able to shown that the light intensity from the unpolarized light source is decreasing and increasing periodically as it was rotated continuously. At different angle, the Polaroid lenses only allow least amount of light directions to pass through and at some other angles, it allow larger number of light direction to pass through. Hence, the rotation of the polarizer will produce the diminishing and increasing light intensity.

The polarizer tube constructed is also efficient enough to shows polarization axis of LCD computer screen and the calculator LCD screen. At certain rotation of the polarizer, the image of the LCD is clearly seen and there is also a 
rotation angle which dark image was seen. This is because the polarizer is now perpendicular to the polarization direction of the screen. The rotation angle that produces the brightest image is representing that the polarization direction of the LCD is parallel with the polarizer.

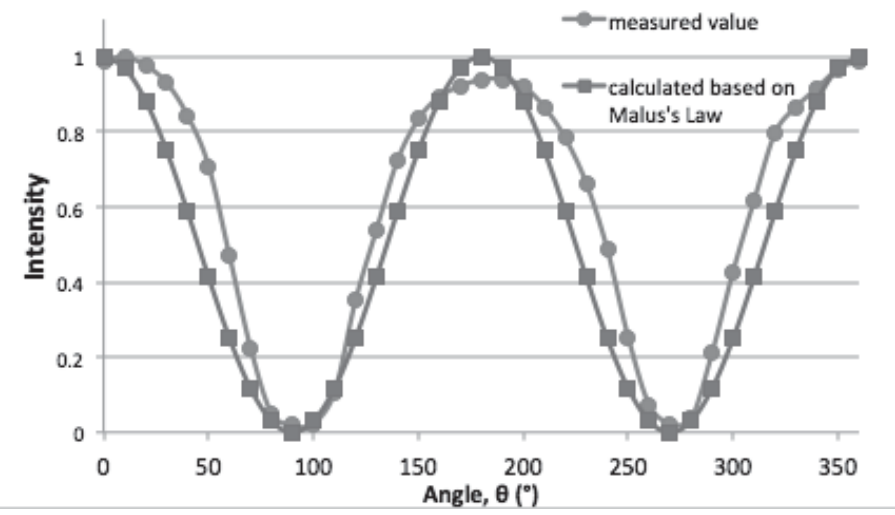

Figure 10: Graph comparison using measured data and calculated value according to Malus's Law.

Polarization property of light allow us to block any direction of light across it and produces dark image. Qualitatively analyzing about polarization will make it easier to understand the basic of polarization. Quantitative analysis of light polarization is very helpful for student to understand about Malus's Law. Traditional teaching by just introducing the Malus's Law and its application is not longer favorable in modern teaching. Student understanding can not sustain longer if Malus's Law is taught without any practical consideration. They will just memorize the formula deducted by Malus and applied it into question. Hence, it will be better to let student explore and find out the deduction of Malus's Law by collecting numerical data from a model.

A set of data was collected and compared to the normalized intensity $\left(\cos ^{2} \theta\right)$ as shown in Figure 11 . The experimental data collected had shown to obey the Malus's Law pattern. Slight discrepancy between experimental data and calculated data are possibly due to imperfection in the polarizers and assumption that the change in LDR resistance is linearly proportional to the light intensity.

\subsection{SUMMARY}

We described simple student activity to carry out demonstration in polarization qualitatively and quantitatively using easily and affordable materials. Students do not only learn about light polarization but integrate the knowledge of electricity and electronics in the activity.

\subsection{REFERENCES}

[1] Malaysian Examinations Council," Malaysia Higher School Certificate Examination” 6 May 2013. http://www.mpm.edu.my/web/guest/sukatan-pelajaran-stpm-modular

[2] University of Cambridge International Examinations., "Cambridge International A \& AS Level Physics, Syllabus code 9702 For Examination in June and November 2013," 6 May 2013. www.cie.org.uk

[3] Ashok., P. C., James, J., Krisha, Y., Chacko., V, Nampoori., V.P.N, "Development of Optics Kit for Schools in Developing Contries - International School of Photonics Model", Proc. ETOP 2009, North Wales, UK, July $5^{\text {th }}-7$ th (2009).

[4] Cutnell, J. D and Johnson, K.W., [Physics 5th Ed.], John Wiley, New York, 746-753 (2001) 


\section{APPENDIX Hands-on Activity}

\section{Activity 1 : Light can change resistance}

Material Light Dependent Resistor (LDR), multimeter, light from lamp in your classroom, a piece of face tissue

Student will

- measure resistance of LDR.

- observe that resistance of the LDR depends on the light intensity onto it.

Procedure

1. Connect each probe of the multimeter to each end of the LDR.

2. Turn the multimeter knob to resistance measurement mode.

3. Direct LDR active area to the light. Measure and record the resistance.

4. Cover the LDT active area with a piece of face tissue. Measure and record the resistance.

5. Cover the LDT active area with your thumb. Measure and record the resistance.

6. Compare the resistance measured in Step 4,5 and 6. What is the relation between light intensity and the resistance of the LDR?

\section{Activity 2: Light detector}

Material Same as the Activity 1 with additional $2.2 \mathrm{M} \Omega$ resistor, wire and $3 \mathrm{~V}$ battery with holder.

Student will

- build the circuit of voltage divider

- build simple light detector.

Procedure

1. Connect each components according to the Fig 1.

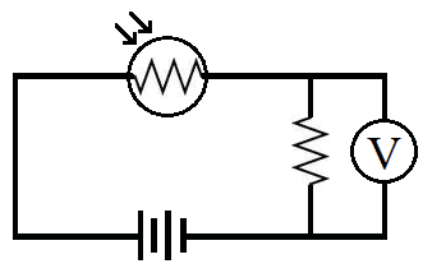

Figure $1 \quad$ Light detector

2. Turn the multimeter knob to the direct voltage measurement mode.

3. Direct LDR active area to the light. Measure and record the resistance.

4. Cover the LDR active area with a piece of face tissue. Measure and record the voltage

5. Cover the LDR active area with your thumb. Measure and record the voltage.

6. Compare the resistance measured in Step 4, 5 and 6.

7. What is the relation between light intensity and the voltage across the LDR? 


\section{Activity 3: Light source}

Material Light Emitting Diode (LED), 3 volt battery with holder, resistor, $120 \Omega$ resistor.

Student will

- build simple light source using LED.

- know that certain electronic components has electrical polarity.

Procedure

1. Connect each components according to the Fig 1.

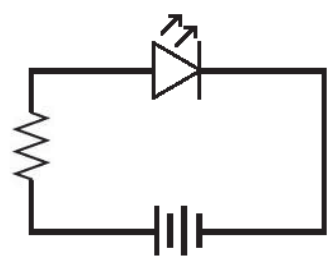

2. Is there any difference when you exchange the leg of the resistor?

3. Is there any difference when you exchange the leg of the resistor?

\section{Activity 4: Linear Polarizer Axis}

Material Linear polarizer, glare or reflection from any flat surface

Student will

- determine the polarization axis of a polarizer

Procedure

1. Look around you and find out any reflection from flat surface for example on glass or water surface.

2. Look the reflection through your polarizer.

3. Rotate and observe any change in your view on the reflection.

4. Mark a line parallel to the horizontal on your polarizer where the seen reflection is minimized.

\section{Activity $5 \quad$ Polarization is everywhere}

Material Linear polarizer, digital display devices (LCD screen, calculator, arm watch)

Student will

- notice that modern devices emits polarized light

Procedure

1. Look into any digital display devices through your polarizer.

2. Rotate the polarizer and notice any change in the view.

3. Could you determine the polarization direction of the emitted light from these devices? 
Material two linear polarizers with known polarization axis, pipe tubes with two close diameter size, glue, cutter

Student will

- observe intensity variation when two polarizers are set at different polarization axes.

- observe intensity minima at cross polarizers

- observe Intensity maxima when polarizers axes are parallel.

Procedure

1. Cut pipe tubes so that the smaller diameter tube can be rotated inside the larger diameter tube

2. Cut polarizers into circular shape which fit the inner diameter of pipe tubes.

3. Glue the polarizers into the tube. Allow to dry.

4. Look into any source of light (outside of the window, pendarfluor light, etc) through these polarizers.

5. Rotate the one polarizer relative to the other one and notice any change in the intensity.

6. At what condition the light intensity is maximum?

7. At what condition the light intensity is minimum.

\section{Activity $7 \quad$ Malus's Law of Polarization}

Material Light detector from Activity 2, Light source from Activity 3, Polarizer tubes from Activity 6 and 2 Tube caps

Student will

- experimentally prove the intensity of light passing through polarizers follow Malus's Law of Polarization

Procedure

1. Assemble light detector and light source at the inner side of each the tube caps.

2. Attach all light detector, light source and polarizer tube together to form observation tube. Make sure no light can enter the inner observation tube.

3. Set the angle between polarization axes at $0^{\circ}$. Note the measured voltage as $V_{o}$.

4. Measure the voltage at different angle from $0^{\circ}$ to $360^{\circ}$ at $10^{\circ}$ increment and fill in Table 1.

Table 1

\begin{tabular}{|c|c|c|c|}
\hline$\theta\left({ }^{\circ}\right)$ & $V_{m}(V)$ & $\begin{array}{c}\text { Normalized Voltage, } \\
V_{N}=V_{m} / V_{O}\end{array}$ & $\begin{array}{c}\text { Normalized Intensity, } \\
\operatorname{Cos}^{2} \theta\end{array}$ \\
\hline 0 & & & \\
\hline 10 & & & \\
\hline 20 & & & \\
\hline. & & & \\
\hline
\end{tabular}

5. Plot the normalized voltage and normalized intensity versus $\theta$.

6. Compare between the two plots. Report you observation. 